\title{
Synergistic Suppression of Cortical Spreading Depression under NR2A and NR2B Inhibition
}

\author{
Yiman Jia, Jiayi Zhou, Fan Bu, Minyan Wang* \\ Centre for Neuroscience, Department of Biological Sciences, Xi'an Jiaotong-Liverpool University, Suzhou, China \\ Email: *minyan.wang@xjtlu.edu.cn
}

Received 18 November 2015; accepted 22 December 2015; published 25 December 2015

Copyright (C) 2015 by authors and Scientific Research Publishing Inc.

This work is licensed under the Creative Commons Attribution International License (CC BY).

http://creativecommons.org/licenses/by/4.0/

(c) (i) Open Access

\begin{abstract}
Cortical spreading depression (CSD) is a transient synaptic excitation, followed by depression, which can propagate slowly across cortex, subcortex and retina. CSD is implicated in migraine with aura and may lead to migraine pain. CSD can be suppressed by inhibition of both NR2A and NR2B containing N-methyl-D-aspartic acid (NMDA) receptors. However, whether there is a synergistic effect of co-inhibition of NR2A and NR2B containing receptors on CSD remains unknown. In this study, an efficient in vitro model of migraine with intrinsic optical imaging approach was applied to address this role of co-activation of both NR2A and NR2B in CSD. The results showed that co-application of NVP-AAM077, a drug selectively targeting for NR2A and Ro 25-6981, a drug selectively targeting for NR2B containing receptors, showed synergistic inhibitory effects on propagation rate and the magnitude of CSD in a concentration dependent manner in chick retina. Inhibition of CSD propagation was also observed by a clinical acceptable drug that antagonizes both NR2A and NR2B containing receptors, memantine, at $10 \mu \mathrm{M}$. These data suggest that combinational use of antagonists selectively targeting NR2A and NR2B containing receptors could be a useful strategy for preventative treatment of migraine with improved efficacy with potential alleviated side effects.
\end{abstract}

\section{Keywords}

Cortical Spreading Depression, NR2A, NR2B, NVP-AAM077, Ro 25-6981, Memantine, Chick Retina

\section{Introduction}

Cortical spreading depression (CSD) is a temporary propagating synaptic excitation, followed by depression, which can propagate across cerebral cortex, subcortical regions and retina. CSD is implicated in migraine with

\footnotetext{
${ }^{*}$ Corresponding author.
}

How to cite this paper: Jia, Y.M., Zhou, J.Y., Bu, F. and Wang, M. (2015) Synergistic Suppression of Cortical Spreading Depression under NR2A and NR2B Inhibition. Pharmacology \& Pharmacy, 6, 573-579. 
aura [1] and may lead to sensitization of migraine pain pathway [2] [3] via both central [4] and peripheral modulations [5] [6]. CSD is accompanied with massive release of neurotransmitters, peptides and cerebral blood flow changes and can also be mediated by key neurotransmitter receptors [7] [8] or neuropeptides [9] [10], pointing them possible therapeutic targets in migraine prophylaxis. Consequently, CSD has been regarded as a useful tool for testing current and novel prophylactic anti-migraine agents [11].

N-methyl-D-aspartic acid (NMDA) receptors have been recognized as a crucial target against CSD elicitation and propagation as MK801, a nonsubtype-selective channel blocker [12], shows strong but irreversible suppressive effects on CSD propagation [7] [13]. However, this agent was clinically unacceptable due to its blockade of normal brain function resulting from complete blockade of the channel. NMDA receptors are heteromeric tetramer consisting of two NR1 and two of four NR2 (A-D) and/or one of NR3 (A-B) subunits [14]. Previous studies showed that NR2A- or NR2B-selective NMDA antagonists can partially suppresses CSD both in vitro [8] or in vivo [13], however with lower efficacy than MK801 [8] [13]. Interestingly, memantine, an non-competitive lowaffinity NMDA receptor channel blocker, first synthesized by Eli Lilly and Company in 1968, which targets both NR2A and NR2B subunits, is clinically well tolerated for treating moderate to severe migraine [15]-[17] and the drug was shown to have anti-CSD effect in rats [13]. We therefore speculate that drugs selectively antagonizing both N2A and NR2B subunits receptor may have greater potential for therapeutic application with improved efficacy and alleviated side effects than competitive channel blockers.

To test this hypothesis, we applied an in vitro efficient CSD migraine model in chick retina complemented with intrinsic optical imaging approach to address synergistic effects of co-application of NR2A-selective and NR2B-selective NMDA receptor antagonists on the magnitude of CSD and propagation rate. The role of these subunits in CSD propagation was also confirmed by memantine in the same tissue.

\section{Methods}

Twenty seven male chicks (WuXi Yangzichang Ltd., Wuxi, China) were housed for at least a week before use (aged 8 - 28 days). Chick retina preparation from posterior eyecup was as that described previously [18]. All animal procedures were approved by the Ethical Review Panels of Soochow University under agreement with Xi'an Jiaotong-Liverpool University and performed in accordance with the associated guidelines. Efforts were made to minimize animal suffering to reduce animal use.

\subsection{CSD Induction in Chick Retina with Intrinsic Optical Imaging}

Tissue preparation and CSD induction were as that described previously [18]. Briefly, posterior eyecups were positioned in a chamber with temperature kept constant at $32^{\circ} \mathrm{C}$. Unless otherwise stated, the chamber was perfused at $0.5 \mathrm{ml} / \mathrm{minute}$ with Ringer's solution (concentrations in mM: $100 \mathrm{NaCl}, 6 \mathrm{KCl}, 1 \mathrm{MgSO}_{4}, 30 \mathrm{NaHCO}_{3}$, $1 \mathrm{NaH}_{2} \mathrm{PO}_{3}, 1 \mathrm{CaCl}_{2}$ and 20 glucose; bubbled with 95\% $\mathrm{O}_{2}$ and 5\% $\mathrm{CO}_{2} ; \mathrm{pH} 7.3, \mathrm{NaCl}$ was purchased from Fisher Scientific and all other reagents were from Sigma-Aldrich). The tissue was stabilized for at least $30 \mathrm{mi}-$ nutes before elicitation of the first CSD episode. Ten repeated CSD episodes were induced by $0.1 \mathrm{mM} \mathrm{KCl} \mathrm{with}$ 20-minute interval for tissue recovery. The retina was illuminated for 25 ms every $1 \mathrm{~Hz}$ using a high-power LED spotlight (SLS-0307-A, Mightex; Pleasanton, CA, USA) and the illumination was driven by a computer-controlled power supply (Sirius LED controller, SLC-SA04-U; Mightex, Pleasanton, USA). The reflected light was simultaneously recorded with a monochrome camera (QIC-F-M-12, Media Cybernetics, Marlow, UK) used at a maximal spatial resolution. Image sequences were taken at $1 \mathrm{~Hz}$ over a 3-minute period, started as CSD was elicited. Camera exposure and illumination were synchronized using the same external trigger (TG5011, TTi, UK). Image Pro Plus software (IPP 7.0; Media Cybernetics, China) was used for image acquisition, storage and analysis.

\subsection{Experimental Design for Drug Testing}

To investigate effects of NVP-AAM077 in the absence or presence of Ro 25-6981, and effects of memantine on CSD magnitude and tissue excitability to CSD in chick retina, four groups were designed: 1) memantine hydrochloride (1-amino-3,5-dimethyladamantane hydrochloride) (Sigma, China) at 1, 3, $10 \mu \mathrm{M}(n=7)[19]$; 2) a NR2A selective NMDA receptor antagonist, NVP-AAM077 ([[[(1S)-1-(4-Bromophenyl)ethyl]amino](1,2,3,4tetrahydro-2,3-dioxo-5-quinoxalinyl)methyl] phosphonic acid) alone, at 0.03, 0.1, $0.3 \mu \mathrm{M}(n=7)$ [20]; 3) NVP- 
AAM077 (0.03, 0.1, $0.3 \mu \mathrm{M})$ and a NR2B selective antagonist, Ro 25-6981 ([R-(R*,S*)]- $\alpha$-(4-Hydroxyphenyl)$\beta$-methyl-4-(phenylmethyl)-1-piperidinepropanol) [2] at 1, 3, $10 \mu \mathrm{M}(n=6)$; (iv) Ringer's solution $(n=7)$. For each group tested, ten repeated CSDs were induced in each experiment, with two separate CSDs for each of the different and consecutive tests (Figure 1): 1) initial Ringer's control; 2) low concentration of drug or vehicle; 3) medium concentration of drug or vehicle; 4) high concentration of drug of vehicle; 5) post-treatment with Ringer's control (i.e. drug removal). For each test sequence, the perfusion medium was changed immediately after the end of the $2^{\text {nd }}, 4^{\text {th }}, 6^{\text {th }}$, and $8^{\text {th }}$ CSD recording when required, such that the preparation was adequately perfused with proper drug or vehicle for subsequent test (Figure 1).

\subsection{Data Presentation and Statistical Analysis}

As reported previously [8], for each 360-frame sequence, an area of interest (AOI) parallel to each CSD wave front was delineated manually (Figure 2). For each picture within the sequence, the gray levels of the pixels constituting the AOI were averaged and plotted against the time as an indicator to characterize the excitation phase of CSD (Figure 2). For each CSD wave, the area under the curve (AUC, gray levels $\times$ minute) of the transient cellular depolarization was calculated and used as an index of the magnitude of propagating CSD. For each CSD wave, propagation rate was also calculated to reflect the degree of tissue excitability. The calculated values within each different test were averaged and all corresponding data were given as mean \pm SD in percentages of their respective baselines, that is, averaged value for the first two $\mathrm{K}^{+}$stimuli. Mann-Whitney test was used for comparison of the magnitude and propagation rate of CSD between memantine and control group $\left({ }^{*} p<\right.$ $0.05,{ }^{* *} p<0.01,{ }^{* * * *} p<0.001$ ). Kruskal-Wallis with subsequent Dunn's test was used for comparison of these two parameters among NVP-AAM077, NVP-AAM077 \& Ro 25-6981 and control group $\left({ }^{*} p<0.05,{ }^{* *} p<0.01\right.$, ${ }^{* * *} p<0.001$ ). Paired t-test was used to compare the significance of the difference for the last two tests with each

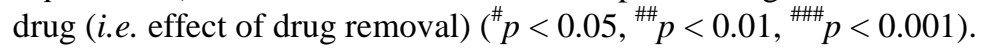

\section{Results}

\subsection{Synergistic Suppression of CSD by Co-Application of NVP-AAM077 and Ro 25-6981}

In the Ringer's group, there was no significant change in CSD magnitude and propagation rate over repeated CSD episodes throughout the experiment (Figure 3, $n=7$ ). NVP-AAM077 alone markedly suppressed both
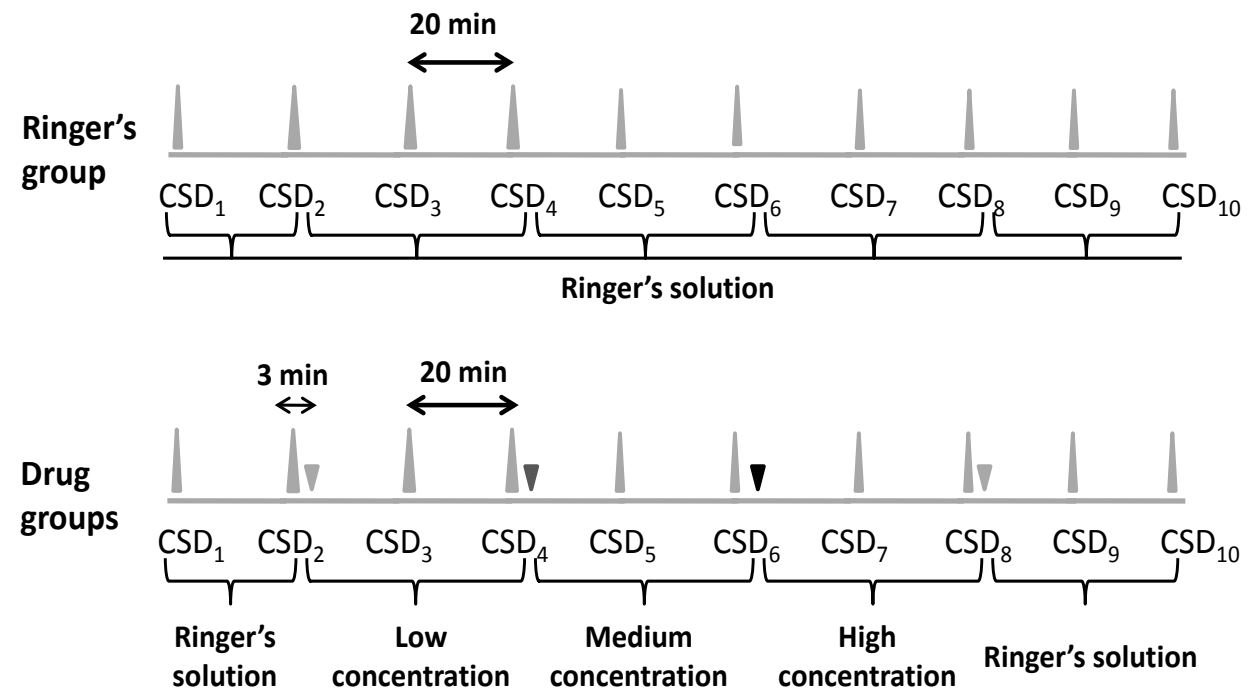

Figure 1. Experimental protocol for investigating effects of drugs targeting NR2A and NR2B containing receptors in the chick retina. Four groups were designed with Ringer's group and three drug groups. In each experiment, ten repeated CSD was induced by high $\mathrm{K}^{+}$-medium, with two separate CSDs for each of the different and consecutive tests. Each CSD wave was followed by 20 minutes perfusion of Ringer's solution for tissue recovery. Each CSD was recorded for 3 minutes at the start of $\mathrm{K}^{+}$-medium perfusion. 

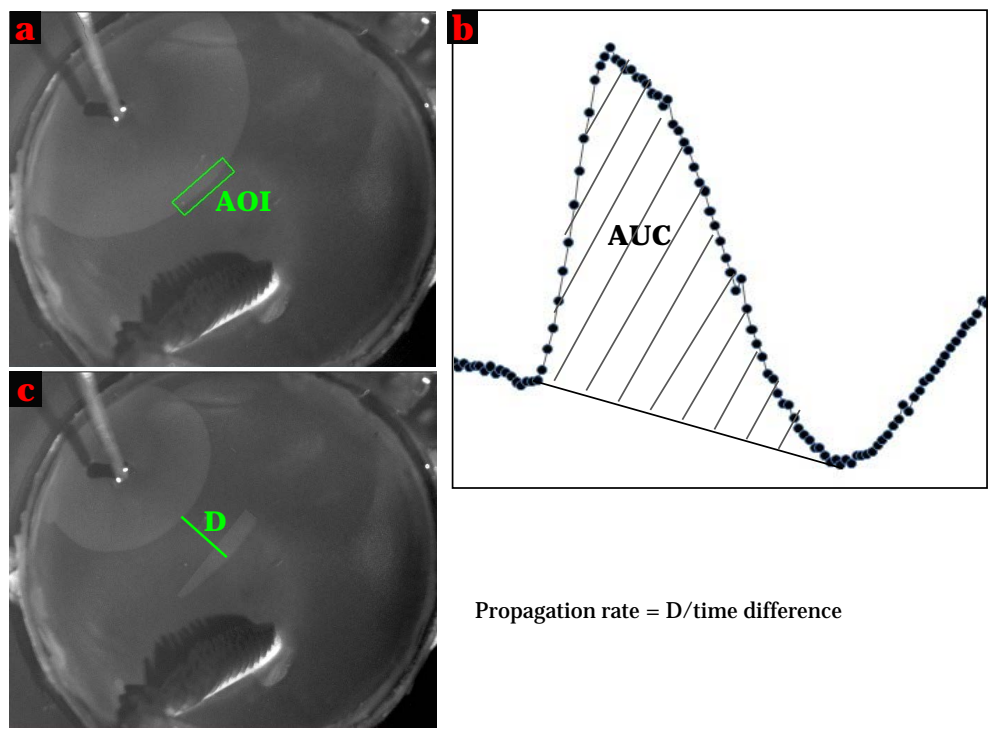

Propagation rate $=\mathrm{D} /$ time difference

Figure 2. Representative image (left, upper panel) and plot of CSD propagation wave (right) induced by high $\mathrm{K}^{+}$-medium in the chick retina. The same area of interest (rectangle within left picture, AOI) was selected and used for all pictures of the sequence under study. All the averaged gray levels within the AOI were plotted against time to generate the CSD wave plot (right). Area under the curve (AUC) of CSD wave front were determined by image analysis to quantify CSD magnitude and propagation rate was calculated as indicated by the distance (D) between two CSD wave fronts within the same AOI at the two different time points over the time duration to quantify drug effects on tissue excitability to CSD.

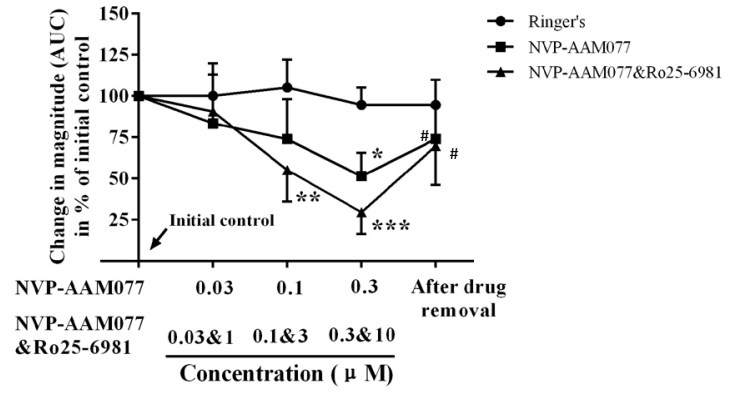

(a)

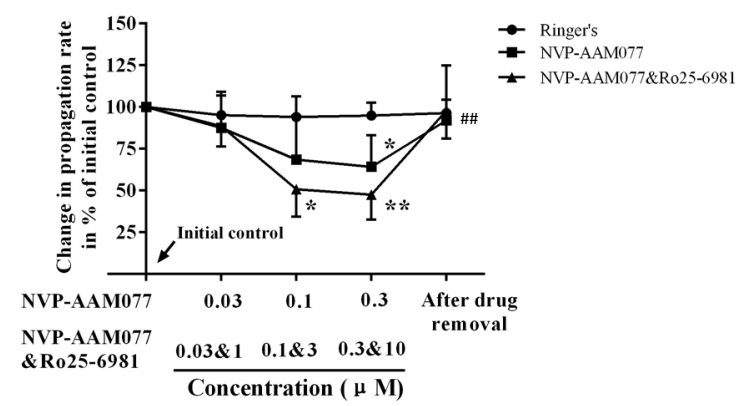

(c)

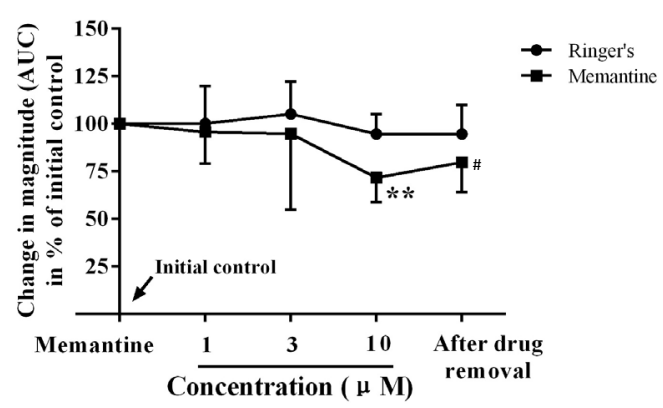

(b)

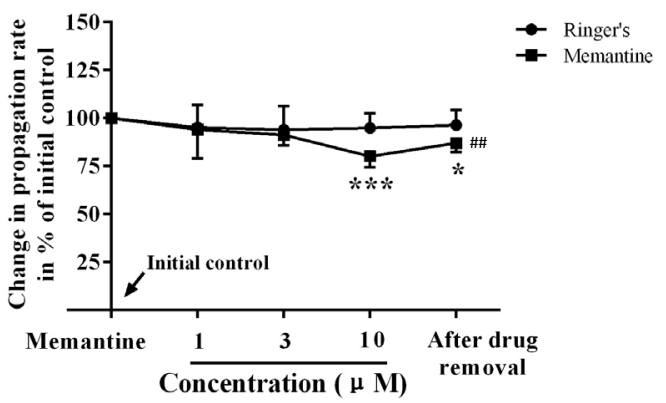

(d)

Figure 3. Effects of NVP-AAM077 in the absence and presence of Ro 256981 and memantine on CSD magnitude as indicated by area under the curve (AUC, 3A and 3C), and propagation rate (3B and 3D) induced by $\mathrm{K}^{+}$in chick retina. Data was plotted as percentage of their initial levels and indicated as mean \pm SD. Mann-Whitney test was used for comparison of the magnitude and propagation rate of CSD between memantine and Ringer's group. Kruskal-Wallis with subsequent Dunn's test was used for comparison of these two parameters among NVP-AAM077, NVP-AAM077 \& Ro 25-6981 and Ringer's group $\left({ }^{*} p<0.05,{ }^{* *} p \leq 0.01,{ }^{* * *} p \leq 0.001\right)$. Paired t-test was used to compare the significance of the difference for the last two tests with each drug (i.e. effect of drug removal, $\left.{ }^{\#} p<0.05,{ }^{\# \#} p \leq 0.01\right)$. 
CSD magnitude (Figure 3(a)) and propagation rate (Figure 3(b)) in a concentration dependent manner when compared with Ringer's group. At the maximum concentration tested $(0.3 \mu \mathrm{M})$, the magnitude and propagation rate of CSD was reduced to 51.43\% (Figure 3(a), ${ }^{*} p<0.05$ ) and $64.17 \%$ (Figure 3(b), ${ }^{*} p<0.05$ ) of initial level respectively. This inhibitory effect recovered to $74.06 \%\left({ }^{\sharp} p<0.05\right)$ and $92.06 \%$ of initial level respectively after the drug removal.

Co-application of NVP-AAM077 and Ro 25-6981 produced a synergistic inhibitory effect with a further reduction of CSD magnitude and propagation rate in a concentration dependent manner (Figure 3(a) and Figure 3(b)). Addition of Ro 25-6981 at the medium concentration applied, $3 \mu \mathrm{M}$, brought the CSD magnitude and propagation rate under NVP-AAM077 application at $0.1 \mu \mathrm{M}$ from insignificance to significance (Figure 3(a) and Figure $3(\mathrm{~b}),{ }^{* *} p<0.01$ and ${ }^{*} p<0.05$ respectively) when compared with the control group. Addition of Ro $25-6981$ at $10 \mu \mathrm{M}$ to NVP-AAM077 at $0.3 \mu \mathrm{M}$ further reduced CSD magnitude and propagation rate to $29.68 \%$ $\left({ }^{* * *} p<0.001\right)$ and $47.55 \%\left({ }^{* * *} p<0.01\right)$ of initial level respectively. This inhibitory effect recovered to $69.78 \%$ $\left({ }^{\#} p<0.05\right)$ and $98.15 \%\left({ }^{\# \#} p<0.01\right)$ of initial level after drug removal (high concentration vs. drug removal).

\subsection{Suppression of CSD by Memantine}

Similarly as NVP-AAM077, memantine reduced CSD magnitude and propagation rate (Figure 3(c) and Figure $3(\mathrm{~d}), n=7)$ when compared with Ringer's group. At the maximum concentration tested $(10 \mu \mathrm{M})$, the AUC and propagation rate was reduced to $71.7 \%\left({ }^{* *} p<0.01\right)$ and $80.21 \%\left({ }^{* * *} p<0.001\right)$ of initial level respectively. These inhibitory effects on AUC and propagation rate slightly recovered to $79.79 \%\left({ }^{\#} p<0.05\right)$ and $87.1 \%\left({ }^{\# \#} p<0.01\right)$ of initial level after drug removal.

\section{Discussion}

The main finding of this work was that co-application of NVP-AAM077 and Ro 25-6981 showed synergistic inhibitory effects on CSD magnitude and propagation rate, suggesting simultaneous inhibition of NR2A- and NR2B-containing NMDA receptors is a useful strategy for migraine prophylaxis with improved efficacy. The additional inhibitory effect by Ro 25-6981 to NVP-AAM077 on tissue excitability and CSD magnitude in chick retina (Figure 3(a) and Figure 3(b)) is in consistent with previous findings that NR2B contributes to CSD propagation both in rats using electrophysiology recording [13], fMRI [21] and intrinsic optical imaging in chick retina [8]. Additionally, this study extended previous findings that inhibition of NR2A alone suppresses tissue excitability to CSD propagation in the same tissue [8]. It was noted that the inhibitory effect on CSD in the chick retina under NR2A inhibition alone by NVP-AAM077 was on contrast to that an unchanged BOLD CSD response was found under NR2A inhibition by TCN-201 [21]. We speculate that this discrepancy may be due to 1) different tissue and species used (rat $v s$ chick retina); and importantly 2) a neuronal mechanism is associated with NR2A mediated CSD in chick retina, a tissue devoid of blood vessel, versus involvement of a vascular component as BOLD signal was dependent on vascular components. Further study is required to clarify the role of NR2A in CSD in vivo.

Memantine was previously shown to inhibit the nociceptive process from trigemino-ophthalmic nerve endings to the trigeminocervical complex in peripheral pain pathway [22] and the drug has efficacy in migraine [15] [16]. In this study, we found a slight but significant suppressive effect by memantine on CSD propagation in chick retina data supporting a neuronal action of NR2A in migraine pathology. This data is compatible with previously published finding in rats [13] and chick retina [23]. As the action of memantine at the concentration applied is associated with both NR2A and NR2B [19], this result supports that both major subunits are required for CSD propagation, which supports our notion that the synergistic effects observed with co-application of the two antagonists targeting NR2A and NR2B.

\section{Conclusion}

In summary, the present study demonstrated a synergistic effect of co-inhibition of NR2A and NR2B containing receptors on CSD in chick retina, suggesting combinational use of selected drugs targeting both the two NR2 subunits may have improved effectiveness in preventive treatment of CSD-associated migraine. Furthermore, both NVP-AAM077 and Ro 25-6981 may have great potential for clinical use against migraine with likely reduced adverse effects, which although requires further investigation in clinic. These data also support that CSD 
in chick retina with intrinsic optical imaging is a useful tool for assessing drugs targeting NMDA receptor for migraine prophylaxis. However, whether combinational efficacy can be repeated in vivo needs further investigation.

\section{Acknowledgements}

This work was partly supported by Wangwenli Charitable Foundation, China and XJTLU research development fund. The authors would thank Junxu Bai for his technical support.

\section{References}

[1] Hadjikhani, N., Sanchez Del Rio, M., Wu, O., Schwartz, D., Bakker, D., Fischl, B., et al. (2001) Mechanisms of Migraine Aura Revealed by Functional MRI in Human Visual Cortex. Proceedings of the National Academy of Sciences USA, 98, 4687-4692. http://dx.doi.org/10.1073/pnas.071582498

[2] Moskowitz, M.A. (2007) Athophysiology of Headache-Past and Present. Headache, 47, S58-S63. http://dx.doi.org/10.1111/j.1526-4610.2007.00678.x

[3] Russo, A.F. (2015) Calcitonin Gene-Related Peptide (CGRP): A New Target for Migraine. Annual Review of Pharmacology and Toxicology, 55, 533-552. http://dx.doi.org/10.1146/annurev-pharmtox-010814-124701

[4] Karatas, H., Erdener, S.E., Gursoy-Ozdemir, Y., Lule, S., Eren-Kocak, E., Sen, Z.D., et al. (2013) Spreading Depression Triggers Headache by Activating Neuronal Panx1 Channels. Science, 339, 1092-1095. http://dx.doi.org/10.1126/science.1231897

[5] Zhang, X., Levy, D., Kainz, V., Noseda, R., Jakubowski, M. and Burstein, R. (2011) Activation of Central Trigeminovascular Neurons by Cortical Spreading Depression. Annals of Neurology, 69, 855-865. http://dx.doi.org/10.1002/ana.22329

[6] Zhang, X., Levy, D., Noseda, R., Kainz, V., Jakubowski, M. and Burstein, R. (2010) Activation of Meningeal Nociceptors by Cortical Spreading Depression: Implications for Migraine with Aura. Journal of Neuroscience, 30, 88078814. http://dx.doi.org/10.1523/JNEUROSCI.0511-10.2010

[7] Marrannes, R., Willems, R., De Prins, E. and Wauquier, A. (1988) Evidence for a Role of the N-Methyl-D-aspartate (NMDA) Receptor in Cortical Spreading Depression in the Rat. Brain Research, 457, 226-240. http://dx.doi.org/10.1016/0006-8993(88)90690-7

[8] Wang, M., Chazot, P.L., Ali, S., Duckett, S.F. and Obrenovitch, T.P. (2012) Effects of NMDA Receptor Antagonists with Different Subtype Selectivities on Retinal Spreading Depression. British Journal of Pharmacology, 165, $235-244$. http://dx.doi.org/10.1111/j.1476-5381.2011.01553.X

[9] Shi, H., Li, J.H., Ji, C.F., Shang, H.Y., Qiu, E.C., Wang, J.J., et al. (2010) Effect of Electroacupuncture on Cortical Spreading Depression and Plasma CGRP and Substance P Contents in Migraine Rats. Zhen Ci Yan Jiu, 35, 17-21.

[10] Tozzi, A., de Iure, A., Di Filippo, M., Costa, C., Caproni, S., Pisani, A., et al. (2012) Critical Role of Calcitonin GeneRelated Peptide Receptors in Cortical Spreading Depression. Proceedings of the National Academy of Sciences USA, 109, 18985-18990.

[11] Costa, C., Tozzi, A., Rainero, I., Cupini, L.M., Calabresi, P., Ayata, C., et al. (2013) Cortical Spreading Depression as a Target for Anti-Migraine Agents. Journal of Headache and Pain, 14, 62. http://dx.doi.org/10.1186/1129-2377-14-62

[12] Chen, H.S. and Lipton, S.A. (2006) The Chemical Biology of Clinically Tolerated NMDA Receptor Antagonists. Journal of Neurochemistry, 97, 1611-1626. http://dx.doi.org/10.1111/j.1471-4159.2006.03991.X

[13] Peeters, M., Gunthorpe, M.J., Strijbos, P.J., Goldsmith, P., Upton, N. and James, M.F. (2007) Effects of Pan- and Subtype-Selective N-Methyl-D-Aspartate Receptor Antagonists on Cortical Spreading Depression in the Rat: Therapeutic Potential for Migraine. Journal of Pharmacology and Experimental Therapeutics, 321, 564-572. http://dx.doi.org/10.1124/jpet.106.117101

[14] Paoletti, P. and Neyton, J. (2007) NMDA Receptor Subunits: Function and Pharmacology. Current Opinion in Pharmacology, 7, 39-47. http://dx.doi.org/10.1016/j.coph.2006.08.011

[15] Bigal, M., Rapoport, A., Sheftell, F., Tepper, D. and Tepper, S. (2008) Memantine in the Preventive Treatment of Refractory Migraine. Headache, 48, 1337-1342. http://dx.doi.org/10.1111/j.1526-4610.2008.01083.x

[16] Krymchantowski, A. and Jevoux, C. (2009) Memantine in the Preventive Treatment for Migraine and Refractory Migraine. Headache, 49, 481-482. http://dx.doi.org/10.1111/j.1526-4610.2009.01349.x

[17] Spengos, K., Theleritis, C. and Paparrigopoulos, T. (2008) Memantine and NMDA Antagonism for Chronic Migraine: A Potentially Novel Therapeutic Approach? Headache, 48, 284-286. http://dx.doi.org/10.1111/j.1526-4610.2007.01016.x 
[18] Wang, M., Li, Y. and Lin, Y. (2015) GABAA Receptor $\alpha 2$ Subtype Activation Suppresses Retinal Spreading Depression. Neuroscience, 298, 137-144. http://dx.doi.org/10.1016/j.neuroscience.2015.04.016

[19] Ogden, K.K. and Traynelis, S.F. (2011) New Advances in NMDA Receptor Pharmacology. Trends in Pharmacological Sciences, 32, 726-733. http://dx.doi.org/10.1016/j.tips.2011.08.003

[20] Wang, M., Chazot, P.L., Ali, S., Duckett, S.F. and Obrenovitch, T.P. (2012) Effects of NMDA Receptor Antagonists with Different Subtype Selectivities on Retinal Spreading Depression. British Journal of Pharmacology, 165, $235-244$. http://dx.doi.org/10.1111/j.1476-5381.2011.01553.x

[21] Shatillo, A., Salo, R.A., Giniatullin, R. and Grohn, O.H. (2015) Involvement of NMDA Receptor Subtypes in Cortical Spreading Depression in Rats Assessed by fMRI. Neuropharmacology, 93c, 164-170. http://dx.doi.org/10.1016/j.neuropharm.2015.01.028

[22] Park, J.W., Suh, G.I., Shin, H.E. and Park, G.E. (2012) Influence of Memantine on Nociceptive Responses of the Trigeminocervical Complex after Formalin Injection. Cephalalgia, 32, 308-316. http://dx.doi.org/10.1177/0333102411435986

[23] Kertesz, S., Kapus, G., Gacsalyi, I. and Levay, G. (2010) Deramciclane Improves Object Recognition in Rats: Potential Role of NMDA Receptors. Pharmacology Biochemistry \& Behavior, 94, 570-574. http://dx.doi.org/10.1016/j.pbb.2009.11.012 